\title{
EL DEBATE SOBRE EL FUTURO DE EUROPA: UNA VISIÓN COMPARADA DE LAS POSICIONES DE LOS JEFES DE ESTADO Y/O DE GOBIERNO Y LAS INSTITUCIONES EUROPEAS
}

\author{
The debate on the future of Europe: A comparative \\ vision of the position of the Heads of State and/or \\ Government and European institutions
}

\author{
FABIO GARCÍA LUPATO \\ Universidad Complutense de Madrid \\ fglupato@ucm.es
}

Cómo citar/Citation

García Lupato, F. (2020).

El debate sobre el futuro de Europa: una visión comparada de las posiciones de los jefes de Estado y/o de Gobierno y las instituciones europeas.

Revista de Estudios Políticos, 190, 13-41.

doi: https://doi.org/10.18042/cepc/rep.190.01

\section{Resumen}

Durante la última legislatura europea se han multiplicado los debates sobre el futuro de Europa, intensificados por las policrisis experimentadas por la UE. Este artículo compara las propuestas de las dos principales instituciones europeas, Comisión y Parlamento (PE), y las posiciones de los veinte jefes de Estado y/o Gobierno que participaron en los debates sobre el futuro de Europa organizados por el PE en el período 2017-2019. Este análisis del contenido permite observar cuáles son las diversas posiciones, similitudes, diferencias y el potencial de politización de la integración europea. Se destaca la importancia de centrarse tanto en el régimen de políticas como el modelo de gobernanza y su relación. Los resultados muestran profundas diferencias sobre cómo se concibe el futuro de Europa y cómo existen prioridades más centradas en la legitimidad de input mientras que otras enfatizan la legitimidad de output. Las diversas posiciones muestran la relevancia de ciertos debates -Europa de varias velocidades, diferenciación, homogeneidad, unificación política, la lógica instrumental - y su relación con diversas teorías e hipótesis sobre el futuro de la integración. Adicionalmente, este análisis permite comprender cómo puede desarrollarse 
la Conferencia sobre el Futuro de Europa que la nueva Comisión está organizando durante el período 2020-2022.

\title{
Palabras clave
}

Unión Europea; futuro de Europa; politización; crisis; gobernanza; instituciones de la UE; políticas de la UE; integración europea.

\begin{abstract}
The last European legislature has witnessed a growing number of debates on the future of Europe, intensified by the so-called polycrisis experienced by the EU. This article compares the proposals of the two most relevant European institutions, the Commission and the Parliament (EP), and the position of twenty Heads of State and/or Government that participated in the debates on the future of Europe, organized by the EP, between 2017 and 2019. The content analysis of these proposals and interventions allows us to understand their positions, similarities, differences and the potential for politicization of European integration. The importance of focusing on both the policy regime and the governance model and its relationship is also highlighted. The results stress the profound differences on how the future of Europe is conceived, showing us priorities more focused on input legitimacy while others emphasize issues related to output legitimay. The various positions show the relevance of certain debates —multi-speed Europe, differentiation, homogeneity, political unification, instrumental logic - and its relationship with different theories and hypotheses about the future of integration. Additionally, this analysis allows understanding the possible developments of the Conference on the Future of Europe that the new Commission is organizing, during the period 2020-2022.
\end{abstract}

\section{Keywords}

European Union; future of Europe; politicization; crisis; governance; EU institutions; EU policies; European integration. 


\section{SUMARIO}

I. INTRODUCCIÓN. II. POLITIZACIÓN Y LA FORMA DE LA UE DEL FUTURO: 1. La creciente politización de la UE. 2. ¿̇Qué forma tendrá la UE? III. ALGUNAS CONSIDERACIONES METODOLÓGICAS. IV. IDEAS SOBRE EL FUTURO DE EUROPA: LA VISIÓN DE LA COMISIÓN Y EL PARLAMENTO. V. EL DEBATE DE LOS JEFES DE ESTADO Y DE GOBIERNO: 1. El régimen de políticas. 2. El modelo de gobernanza - cómo organizar la UE del futuro. 3. ¿ES NECESARIA UNA REFORMA DE LOS TRATADOS? VI. CONCLUSIÓN. BIBLIOGRAFíA.

\section{INTRODUCCIÓN ${ }^{1}$}

En su discurso de apertura en la sesión plenaria del Parlamento Europeo (PE), la entonces candidata a presidenta de la Comisión Europea, Ursula von der Leyen, señaló su deseo de organizar una conferencia sobre el futuro de Europa a lo largo de 2020, que se prolongará durante dos ańos (Leyen, 2019: 10). Esta propuesta continúa con el debate sobre el futuro de Europa que lleva desarrollándose durante la última década en el seno de la propia UE, sus principales instituciones y líderes ${ }^{2}$. Durante la última legislatura europea (20142019) se han multiplicado los debates e iniciativas. Esto es especialmente relevante porque, tras la crisis de 2008, la importancia y oportunidades de la pertenencia a la UE, pero también sus limitaciones, han quedado patentes para los ciudadanos. Esta crítica a la UE ha proporcionado argumentos a

1 Una versión preliminar de este artículo se presentó en el XIV Congreso de la AECPA. Quiero agradecer sus observaciones a la coordinadora del grupo de trabajo, Ruth Ferrero Turrión, y al comentarista, Luis Bouza García. Una segunda versión se presentó en el seminario de otońo del Departamento de Ciencia Política y de la Administración de la UCM y se benefició de comentarios de los asistentes. Agradezco también las útiles observaciones de dos revisores anónimos de la revista. Los errores, en su caso, son responsabilidad del autor.

2 Como el Informe de los cuatro presidentes (Van Rompuy, 2012), Informe de los cinco presidentes (Juncker, 2015), ambos dirigidos a completar la Unión Económica y Monetaria. 
líderes y partidos de diverso corte populista, que han hecho de ella uno de sus principales temas de competición política.

La UE ha experimentado lo que se ha venido a denominar una eurocrisis o policrisis (Hutter y Kriesi, 2019; Zeitlin et al., 2019; Fabbrini y Schmidt, 2019), que incluye la crisis de la eurozona, la crisis de refugiados, el brexit y la crisis de las propias instituciones europeas, mostrando que el proyecto de integración, caracterizado por el consenso, comienza a estar cada vez más politizado. Esta politización puede tener diversas consecuencias — existe un debate abierto sobre ello-, pero indica cómo la propia UE se encuentra en un momento de reflexión sobre su propio futuro.

Unido a esto, y es una novedad, cada vez hay más líderes políticos de partidos relevantes, incluso en el Gobierno - tanto como partidos principales o como socios en Gobiernos de coalición-, con posiciones claramente críticas con respecto a la UE. El euroescepticismo, que era visto como políticas de oposición o de partidos en los márgenes de los sistemas partidistas (Taggart 1998; Sitter, 2002, Hooghe et al., 2002; De Vries y Edwards, 2009) ha llegado al Gobierno. Consecuentemente, representantes de estos partidos forman parte del Consejo Europeo y tienen cada vez mayor peso en el PE, que está experimentando la proliferación de partidos y grupos de naturaleza crítica con la UE.

Este contexto nos muestra la relevancia y actualidad del debate sobre el futuro de la UE. ¿Qué debates se están produciendo? ¿Qué propuestas? ¿Qué puntos de acuerdo y desacuerdo se están cristalizando? En definitiva, ¿cómo es el debate sobre el futuro de Europa?

Un aspecto adicional hace su estudio aún más relevante. Durante los dos últimos años, las principales instituciones se han manifestado, de un modo u otro, sobre esta cuestión (Comisión Europea, 2017; Parlamento Europeo, 2017, 2019; Consejo Europeo, 2019). Junto a ellas, el propio PE organizó una serie de debates entre 2018 y 2019 para escuchar las propuestas e ideas de los jefes de Estado y de Gobierno de los Estados miembros (EE. MM.). Estos debates son especialmente interesantes porque nos muestran las posiciones de los máximos representantes de la soberanía nacional en la sede de la soberanía europea, permitiéndonos observar cuáles son las dinámicas y posiciones de los diversos actores del sistema político europeo. Un aspecto central de esta discusión consiste en la indefinición, incertidumbre y diversas propuestas sobre hacia dónde y cómo debería ser el futuro de la UE. A su vez, todos reconocen la centralidad y relevancia de esta discusión. Es decir, están presentes muchos de los elementos necesarios para la politización.

El objetivo de este artículo consiste en realizar un análisis preliminar de los discursos y propuestas de los principales líderes nacionales en el PE, así como de la Comisión y el PE sobre el futuro de Europa. Conocer estas 
posiciones nos permitirá saber cuáles son los puntos en común y, sobre todo, las divergencias entre las principales instituciones y líderes europeos ${ }^{3}$.

Para analizarlo, este artículo se estructura de la siguiente manera. A continuación, se realizará una revisión bibliográfica sobre la idea de politización de la UE, así como el debate sobre las posibles formas de la UE en el futuro. El tercer apartado presenta algunas consideraciones metodológicas e identifica los principales factores que se utilizarán para codificar las posiciones de las instituciones europeas y de los líderes políticos. El cuarto apartado analiza las propuestas y posiciones de la Comisión y el PE y, posteriormente, se examinarán las intervenciones de los jefes de Estado y/o Gobierno. Por último, se extraerán las conclusiones.

\section{POLITIZACIÓN Y LA FORMA DE LA UE DEL FUTURO}

En este apartado se tratarán dos temas críticos para el análisis del futuro de Europa. Por un lado, la idea de politización de la esfera europea y sus consecuencias. Además, el debate supone plantear, de nuevo, la forma que debería tener la nueva UE.

\section{LA CRECIENTE POLITIZACIÓN DE LA UE}

Como sancionaron hace una década Hooghe y Marks (2009), la UE ha pasado del consenso permisivo al «disenso limitante». La UE, el proceso de integración y sus políticas, pasó de ser percibida como algo lejano, sin impacto directo en los ciudadanos, a empezar a ser mucho más problemática. La crisis de 2008 tuvo gran parte de la culpa, aunque los procesos de creciente integración, con el euro a la cabeza, ya habían comenzado a levantar ciertas suspicacias. Esta politización, indica Schmidt (2019a), se ha producido en diversos niveles. Se ha desarrollado «abajo», a nivel nacional, con el incremento de la polarización en los debates, con electorados divididos, el declive del apoyo a los partidos tradicionales y el auge de partidos euroescépticos. También de «abajo a arriba», con un creciente peso de la política nacional en la posición de los líderes en el Consejo Europeo, en la lógica del constraining dissensus. Finalmente, también «arriba», a nivel de las propias instituciones europeas.

3 El Consejo Europeo (2019), en su Declaración de Sibiu de 9 de mayo también presentó diez propuestas sobre le futuro de Europa, pero no van más allá de compromisos genéricos sobre la unidad, buscar soluciones conjuntas, defender el modo de vida europeo o ser líder mundial responsable. 
Entonces, ¿qué es la politización y cómo afecta al sistema político europeo? Podemos distinguir diversos aspectos. Con respecto a la politización europea, esta ha sido identificada como «el proceso que describe el aumento de la contestación visiblemente pública relacionada con las varias dimensiones de la integración europea» (Hutter y Kriesi, 2019: 2) o «la creciente relevancia, polarización de la opinión y expansión de los actores y audiencias involucradas en los temas de la UE» (De Wilde et al., 2016: 3). Por tanto, es relevante tanto la visibilidad del tema como la existencia de posiciones distintas y contrapuestas, así como actores que compitan en estas cuestiones y sean capaces de movilizar a diversos colectivos.

Sus consecuencias son discutidas. Para unos, es una etapa inevitable en la transformación de los sistemas políticos nacionales y el europeo, con el desarrollo de una nueva fractura (Hooghe y Marks, 2018; Zeitlin et al., 2019). Para otros, en cambio, supone una gran limitación en la capacidad institucional de la UE —al ser un sistema basado en el consenso-, puesto que la idea de fondo es que era necesario despolitizar ciertos temas para llegar a mejores resultados (ibid.: 2-3, para una discusión; De Wilde 2019: 1207). Así, «la UE ha creado múltiples espacios de politización, tanto a nivel nacional como europeo, que han sido ocupados por diversos partidos y movimientos en diferentes países y regiones» (Zeitlin et al., 2019: 3). Ahora tenemos a países deudores y acreedores, países de llegada y de acogida de inmigrantes y asilo, los que quieren diversos tipos de integración y, además, con características especiales por las diversas dimensiones de competición a nivel nacional.

Por último, frente a la idea de que las crisis y la politización pueden poner en riesgo el proceso de integración europea, Jabko y Luhman (2019: 1040) señalan que no es necesariamente así puesto que las crisis pueden convertirse en una ventaja política para los actores proeuropeos. En una línea similar, De Wilde (2019: 1207) considera que «no hay que sobreestimar la politización de la integración europea», debido a que puede ayudar a incrementar la presión para llegar a acuerdos, excepto cuando su relevancia es común y sincrónica en todos los EE. MM. En estos casos, facilita la priorización de los intereses nacionales. De ahí la relevancia de estos debates sobre el futuro de Europa.

La politización a nivel nacional muestra como la polarización y problematización de la UE ha aumentado de distinta manera según los países. La propia Schmidt comentaba ya en 2006 que las formulaciones a nivel nacional se caracterizaban por ser "politics without policies», mientras que en la UE ocurría justo a la inversa. Esto puede haber cambiado. La europeización de las políticas nacionales, como la crisis económica y de inmigración han puesto de relieve, ha llevado a la politización de la propia UE. Ahora bien, esta adquiere diversos matices según el país o región que analicemos y, además, es 
dependiente de actores políticos concretos (Hutter y Kriesi, 2019: 2). Entonces, ¿qué actores politizan la UE? Tradicionalmente, los partidos establecidos primaron las estrategias de despolitización dejando libre el espacio a los partidos en los márgenes del sistema, que han tendido a posicionarse más claramente con argumentos más o menos euroescépticos, ya sea como una piedra de toque del disenso (Taggart, 1998), como políticas de oposición (Sitter, 2002) o siguiendo la idea de curva invertida (Hooghe et al., 2002). Son este tipo de challenger parties euroescépticos «los que se han mostrado como los principales vectores de la politización» (Hutter y Kriesi, 2019: 5), con el añadido de que ahora tiene un mucho mejor resultado electoral e incluso llegan al Gobierno (Zulianello, 2019 sobre los partidos populistas).

En segundo lugar, la politización y su relevancia es muy dependiente de la región europea, especialmente por el impacto de las dos principales crisis: la económica y la de inmigración. En este sentido, existen perspectivas similares. Hutter y Kriesi formulan una hipótesis de politización diferencial. Según su análisis, la integración europea está poco politizada a nivel electoral en Europa Central y del Este, mientras que la eurocrisis mostró un gran crecimiento de la politización en el Sur de Europa, especialmente en Grecia y mucho menos en España; en cambio, la crisis de inmigración fue decisiva en los países del Noroeste de Europa. De manera similar, Hooghe y Marks (2018: 110) han señalado la emergencia de un clivaje transnacional basado en las reacciones políticas contra la integración europea y la inmigración, que tiene su "punto focal en la defensa de los modos de vida políticos, sociales y económicos nacionales frente a actores externos que penetran en el estado a través de las migraciones, el intercambio de bienes o ejerciendo autoridad».

Este nuevo clivaje también muestra patrones territoriales diferenciados. En el Norte de Europa, la respuesta del sistema de partidos y la competición política ha facilitado el surgimiento de partidos de extrema izquierda y de extrema derecha. En el Este de Europa, la respuesta predominante muestra la emergencia o fortalecimiento de partidos de derecha radical, con una extrema izquierda débil o inexistente que, además, ha asumido los valores tradicionales. En el Sur de Europa, en cambio, la repuesta a la crisis del euro y la austeridad cristalizó en el auge de la izquierda radical, toda vez que los partidos de derecha ya tenían valores muy tradicionales. A pesar de ello, esta excepcionalidad del Sur de Europa parece haber finalizado con la emergencia o fortalecimiento de partidos de derecha radical como la Lega o Fratelli d'Italia (Italia), Vox (España) o Chega! (Portugal). Como señala Schmidt (2019a: 1019, énfasis en el original), esta politización "se habría movido del nivel nacional "politics without policy" hacia "politics against policy"». Estas dinámicas de politización nacional también tienen su efecto en las instituciones europeas, en el Consejo o el PE, puesto que «la Comisión, el BCE y un creciente número 
de instituciones reguladoras se han dado cuenta de la importancia política de las percepciones públicas sobre sus acciones». (id.).

Esta creciente importancia de las percepciones públicas es analizada por De Wilde (2019). Aunque su foco se centra más en las lógicas por la cual los medios de comunicación dan cobertura a las instituciones, políticas y debates europeos, los mecanismos que identifica son relevantes para nuestro análisis. Concluye que las dinámicas de cobertura mediática y su impacto en la integración europea favorecen el desarrollo del «intergubernamentalismo discursivo», que supone la primacía de "los actores ejecutivos, particularmente los primeros ministros y jefes de Estado", sus conflictos y debates, también en el Consejo, como el foco más relevante de cobertura (ibid.: 1194). Son los jefes de Estado y de Gobierno los que tienen una mayor y creciente capacidad para dar forma al debate europeo en la esfera pública, con el incentivo para «reconstruir la identidad nacional como el marco de referencia para lo ciudadanos» (ibid.: 1208). Esto puede tener distintos efectos en la politización de la UE. Por un lado, puede complicar los acuerdos entre los diversos países y que, por ejemplo, los líderes rehúyan la reforma de los Tratados como un método para gestionar los problemas europeos. A su vez, puede incrementar la presión para llegar a acuerdos, excepto si los temas europeos son relevantes al mismo tiempo en todos los países. (ibid.: 1208). Esto podría suponer ciertos problemas a la hora de llegar a acuerdos en las discusiones sobre el futuro de Europa, que serán relevantes y coincidentes en el tiempo en todos los EE. MM.

A pesar de la creciente primacía de los Gobiernos y el creciente peso de la lógica nacional, también las instituciones europeas y sus principales líderes poseen mecanismos a su disposición para ser relevantes y reducir la distancia con la ciudadanía. Estos incluyen la propuesta de políticas que defiendan el interés general con un claro impacto positivo en la ciudadanía (De Wilde lo ejemplifica con la eliminación del roaming en la UE); la hipótesis de emprendimiento supranacional, es decir, cómo ciertas figuras con posiciones ejecutivas relevantes pueden ganar relevancia en momentos de crisis (el caso de Draghi al frente del BCE durante la crisis); o el «desbordamiento democrático", por el cual los argumentos en defensa de una mayor rendición de cuentas y transparencia alcanzan mayor visibilidad. En definitiva, a pesar del creciente peso e influencia de los principales líderes nacionales, también las instituciones y actores europeos tienen diversas estrategias para ganar relevancia.

Estas diferentes estrategias cristalizan, además, en unas relaciones cada vez más politizadas entre las principales instituciones, produciéndose un cambio en los equilibrios de poder entre Comisión, Consejo y PE. Esto puede observarse en el creciente papel de los métodos intergubernamentales y la relevancia de acuerdos e, incluso, de órganos informales con atribuciones efectivas. Se producen conflictos políticos sobre legitimidad, autoridad, el proceso 
de toma de decisiones, etc. El sistema de elección de los principales cargos políticos de la UE es un buen ejemplo. El proceso de Spitzenkandidaten, o "candidato principal», fue el sistema propuesto por el PE para elegir al presidente de la Comisión, por el cual el líder designado del partido con mejor resultado electoral en las elecciones europeas debería acceder al cargo, equiparando estos comicios a los nacionales (Hobolt, 2014; Tilindyte, 2019). Esta mejora en la democracia europea supondría limitar la capacidad de elección y negociación por parte de los jefes de Estado y/o de Gobierno. Fue exitosa en 2014 con la elección de Juncker, a pesar de la oposición de una mayoría de bloque suficiente en el Consejo (De Wilde 2019: 1198-99), pero no funcionó para la elección de von der Leyen. Esta politización entre Consejo y PE quedó patente en el cuanto menos tortuoso proceso de evaluación de los comisarios europeos propuestos.

\section{2. ¿̇QUÉ FORMA TENDRÁ LA UE?}

El debate sobre el futuro de Europa también ha producido la reemergencia de la discusión sobre la forma que debería tener la UE en el futuro, lo que se vino a llamar la naturaleza de la bestia (Risse-Kappen, 1996; Börzel y Risse, 2009). Este debate no es solo académico, sino que se ve reflejado en cierta medida en los diversos escenarios que planteó la Comisión Juncker en su Libro blanco sobre el futuro de Europa (Comisión Europea, 2017). Se discute sobre la forma de la UE del futuro, ya sea como una verdadera unión federal o hasta la posibilidad de regímenes diferenciados, varias velocidades e incluso una Europa a la carta.

Para algunos como Schmidt (2019b), la «integración diferenciada» es el futuro de Europa, con la conformación de una unión de múltiples grupos como único modo posible para dar cabida a las diversas crisis de la UE. La lógica de esta noción parte de la constatación de que dos aspectos son esenciales y configuran la esencia de la Unión, el ideal democrático y el mercado común, mientras «no todos los Estados miembros necesitan proceder juntos al mismo ritmo con un conjunto uniforme de instituciones que convergen en el mismo grupo de políticas». Es aquí donde entra su idea de soft-core y la pertenencia «a una pluralidad de comunidades de políticas, incluyendo la eurozona, seguridad y defensa, Schengen además de algunos acuerdos más especializados». (ibid.: 2).

Además, como hemos indicado, la diferenciación regional es clave. La integración diferenciada puede tener más sentido porque la «integración uniforme se vuelve más difícil cuando las preferencias estatales, dependencias y capacidades divergen" (Schimmelfenning y Winzen, 2020: 4) y, como ellos mismos indican, sigue dos lógicas. Por un lado, la instrumental, con 
motivaciones basadas en el coste-beneficio, el alto coste de adopción de nuevas políticas por parte de los nuevos miembros, así como la preocupación sobre las consecuencias en términos de competición, salarios bajos o cumplimiento administrativo en los países más ricos. En todo caso, suele ser temporal. Por otro lado, la diferenciación constitucional, que queda reflejada en la reforma de los Tratados y que sigue una lógica de soberanía nacional e identidad que fomenta la heterogeneidad en las preferencias sobre la integración (ibid: 4-5).

En estas discusiones sobre la forma, Fabbrini (2019: 2) enfatiza la importancia de discutir no solo los policy regimes, es decir, lo que «la UE hace o debería hacer", sino también aspectos relativos a los modelos de gobernanza, que se centran en el quién y cómo se implementa. Deduce cuatro posibles modelos de gobernanza para el futuro de Europa. Dos de estos modelos parten de concebir la UE como una organización regional —el modelo de gobernanza dual y el de club de gobernanza-, mientras que otros dos se derivan de concebir a la UE como una "federación en construcción» - los modelos de Estado federal o de unión federal-. Este es un aspecto central, como veremos, puesto que nos ayuda a distinguir las diversas propuestas y su alcance según la institución o líder que reflexione sobre el futuro de Europa.

En definitiva, el debate sobre el futuro de Europa se enmarca también en su régimen de políticas y modelo de gobernanza. Esto va unido al proceso de politización y la llegada de partidos con visiones diferentes a posiciones de influencia, tanto a nivel nacional como europeo.

\section{ALGUNAS CONSIDERACIONES METODOLÓGICAS}

Antes de iniciar el análisis propiamente dicho, es necesario presentar y discutir ciertos aspectos metodológicos relativos a la selección de las intervenciones y los conceptos aplicados para el análisis del contenido. Este artículo presente un diseño necesariamente exploratorio. Las posiciones de algunos actores e instituciones no están claramente definidas y, más aún, están sujetas a cambios y adaptaciones según avance el proceso, aún en marcha.

La unidad de análisis son los discursos o resoluciones de los actores e instituciones analizadas. Respecto a la posición de la Comisión, se ha elegido el discurso del presidente Juncker en el estado de la Unión de $2017^{4}$. La ventaja de este sexto escenario es que en él sí existe una toma de posición clara sobre cómo ve el futuro de Europa, mientras que los cinco escenarios

4 Celebrado el 17 de septiembre con el título «Una Europa más unida, fuerte y democrática para 2025». 
anteriores fijaban las distintas posibilidades disponibles ${ }^{5}$. Con respecto al PE se han seleccionado las dos resoluciones aprobadas por la institución sobre la misma materia ${ }^{6}$. Las intervenciones de los líderes políticos corresponden a su discurso inicial en las sesiones plenarias organizadas a tal efecto por el $\mathrm{PE}^{7}$. Celebrados entre enero de 2018 y abril de 2019, contaron con la participación de veinte líderes de los EE.MM. — todos excepto los máximos representantes de Austria, Hungría, Chequia, Lituania, Reino Unido, Bulgaria, Malta y Eslovenia- . Aún así, nuestro análisis puede contar con líderes de países que reflejan las divisiones que, como hemos visto, tienen efecto a la hora de comprender las posiciones sobre la integración europea, incluyendo región, la división este/oeste y el impacto diferencial de las policrisis, tamaño, antigüedad en la adhesión, ideología política e incluso posición sobre el proceso de integración europea. La importancia de estos debates, además, radica en que se producen en una nivel «de abajo a arriba» (Schmidt, 2019a), que sitúa a los máximos representantes de la democracia estatal en la sede europea más democrática y que, en principio, representa al pueblo europeo.

Adicionalmente, todas las propuestas se concentran entre 2017 y 2019, evitando que determinados cambios de contexto profundo tengan un impacto en las posibles propuestas.

Para comparar las diversas posiciones, y derivado de la discusión anterior, se codificarán sus intervenciones y propuestas en base a la operacionalización de tres variables clave: régimen de políticas, modelo de gobernanza y la posición con respecto a la reformas de los Tratados.

Como hemos señalado, el régimen de políticas es una parte crucial para entender las propuestas sobre el futuro de la UE. Podemos distinguir entre aquellos que quieren un mayor rol de la UE en un número más amplio de políticas, tanto las ya establecidas como profundizando en ciertas áreas como inmigración, fronteras, seguridad o políticas sociales e, incluso, nuevas políticas como el mercado digital o la lucha contra el cambio climático. Esto es lo

5 Los escenarios se llaman: 1) Seguir igual; 2) solo el mercado único; 3) los que desean hacer más, hacen más; 4) hacer menos pero de forma más eficiente, y 5) hacer mucho más conjuntamente. Para una comparación entre escenarios, ver Comisión Europea (2017: 29).

6 El 16 de febrero de 2017 y el 13 de febrero de 2019.

7 El formato del debate consistía en una primera intervención del líder político, seguido de una intervención del presidente de la Comisión o algunos de sus comisarios y posterior debate con los eurodiputados. Las intervenciones se hicieron en diversas lenguas, aunque el inglés fue predominante. En otras (italiano, francés), el autor pudo codificarlos en su lengua original. En otros casos, pocos pero relevantes (Grecia, Alemania, Polonia), se tradujo vía Google Translator. 
que hemos llamado más comunitarización. Igualmente, se puede considerar que la UE debe centrarse en una serie de políticas concretas, donde aporta valor añadido y que no debe, por defecto, hacer más y más cosas. Esto es lo que hemos llamado focalización y supone, siguiendo un escenario de la Comisión, hacer menos y hacerlo mejor. Finalmente, podría haber propuestas de reducir al máximo las políticas europeas, limitadas al mercado interior. Esto lo hemos denominado nacionalización.

En segundo lugar, siguiendo a Fabbrini (2019), también es importante el modelo de gobernanza. La UE puede ser concebida como una organización regional o como una federación en construcción. Respecto al primer modelo, podemos distinguir dos tipos. La gobernanza dual es la estructura actual de la gobernanza europea, supranacional para el mercado e intergubernamental para las políticas tradicionalmente asociadas a los Estados. El modelo de club de gobernanza, por su parte, supone el desarrollo de una combinación variable de Estados interesados en participar solo en ciertas políticas, donde la UE les puede aportar valor. Por tanto, incluye la distinción entre homogeneidad y diferenciación, en la línea de la Europa soft-core o de integración diferenciada (Schmidt, 2019b; Schimmelfenning y Winzen, 2020). Respecto al modelo federal en construcción, también podemos distinguir otros dos tipos. El Estado federal implica superar la diferenciación actual de la UE a través de la creación de una organización centralizada y homogénea políticamente. En cambio, la unión federal sigue la lógica de las federaciones de EE. UU. o Suiza, y supone crear una república de muchas repúblicas ya existentes, con separación múltiple de poderes y varios niveles de toma de decisiones.

Finalmente, se codificará la posición de los líderes e instituciones sobre su opinión, favorable o contraria, a la reforma de los Tratados. Esta variable es relevante en tanto en cuanto ciertas propuestas sobre el futuro de la UE, políticas o institucionales, pueden necesitar de un nuevo encaje normativo. Como indica De Wilde (2019), los líderes políticos nacionales y la Comisión Europea suelen ser reacios a contemplar el complejo proceso de reforma de los mismos y el resultado de los últimos referendos sobre temas europeos así lo demuestra. En cambio, prefieren desarrollar los mecanismos existentes o crear instituciones de novo (ibid.: 1201, 1204).

La posición de las instituciones y líderes nacionales se materializa en múltiples intervenciones, debates y decisiones concretas. En el caso de la Comisión y PE, aún así, se codificarán intervenciones y documentos que son fruto de un proceso de reflexión que refleja mejor su posición, al menos en un momento determinado. En cambio, las intervenciones de los líderes nacionales no dejan de presentar limitaciones a la hora de inferir la posición de un Gobierno o un país sobre un tema tan relevante y amplio. Una única intervención, de duración limitada, no permite tratar todos los temas con la 
profundidad suficiente para que la posición quede efectivamente clarificada. Además, y es necesario recalcarlo, en la lógica de politización de abajo a arriba estos debates también van dirigidos, incluso especialmente dirigidos, a cada opinión pública nacional. De hecho, todas las intervenciones dedican más o menos espacio a poner en valor las políticas y éxitos de la actuación de cada Gobierno. Por último, podemos encontrarnos con el problema de la relevancia declarada (Marks et al., 2007), que adaptado a nuestro caso supone que los líderes pueden obviar temas que les resulten especialmente incómodos porque exista desacuerdo interno en su Gobierno o su país o no lo considere políticamente oportuno.

A pesar de todo ello, el análisis de estos debates sí que puede aportarnos diversos argumentos y señalar distintas posiciones entre los líderes políticos y las principales instituciones europeas, que pueden ser relevantes para analizar las bases para la politización según avance el debate en el futuro y proporcionar, adicionalmente, argumentos para algunas de las hipótesis que se han presentado en la discusión teórica e, incluso, generar alguna nueva.

\section{IDEAS SOBRE EL FUTURO DE EUROPA: LA VISIÓN DE LA COMISIÓN Y EL PARLAMENTO}

A continuación analizaremos las principales ideas de la Comisión y el Parlamento Europeo. La tabla 1 compara las distintas posiciones de estas dos instituciones, según las variables que hemos identificado.

Tabla 1. Comparación de las propuestas de la Comisión y el PE sobre el futuro de Europa

\begin{tabular}{lcc}
\hline & $\begin{array}{c}\text { Comisión Europea } \\
\text { (Escenario 6 - Juncker 2017) }\end{array}$ & $\begin{array}{c}\text { Parlamento Europeo } \\
\text { (Resolución 2017 y 2019) }\end{array}$ \\
\hline Régimen de políticas & Más comunitarización & Más comunitarización \\
\hline Modelo de gobernanza & Gobernanza Dual & Estado Federal \\
\hline Reforma Tratados & No & Sí \\
\hline
\end{tabular}

Fuente: elaboración propia.

En el régimen de políticas existen puntos de acuerdo entre ambas instituciones, aunque con matices. Ambas proponen una mayor comunitarización de las políticas, abogando por crecientes competencias europeas en diversas 
áreas y defendiendo una mayor uniformidad e integración y, consecuentemente, reduciendo la complejidad de la UE y desechando la idea de las diversas velocidades. Incluso, institucionalmente, ambas defienden unificar la presidencia del Consejo y de la Comisión, así como reducir o eliminar la unanimidad en la toma de decisiones. En definitiva, las dos se oponen a la idea de Europa a la carta y, consecuentemente, para evitar un sistema complejo, demandan que todos aquellos que cumplen con los criterios establecidos pasen a formar parte de determinados acuerdos, como la adopción del euro o Schengen.

Frente a estas similitudes y acuerdos es interesante destacar sus diferencias, especialmente relativas al modo de tratar el futuro de Europa. Mientras la Comisión se plantea más el régimen de políticas, pero mantiene la arquitectura actual de la Unión (la gobernanza dual) y no considera necesaria la reforma de los Tratados ni las instituciones, el PE sí que quiere debatir y reformar el modelo de gobernanza y el entramado institucional europeo. Vemos aquí la lógica diferente de cada institución ante las crisis europeas, que podríamos distinguir entre la legitimidad de origen y de ejercicio, o como indica Scharpf (2003), legitimidad de input y output.

La Comisión busca recuperar la confianza y apoyo ciudadano a través de políticas que tengan un impacto positivo y claro, es decir, reforzando la legitimidad de output. En la línea de los emprendedores supranacionales (De Wilde, 2019), defiende una mayor visibilidad de la UE y de la Comisión y propone una reducción de la diferenciación, incluyendo la participación de todos los EE. MM. en el mercado único, el euro y el pilar social. En cambio, el PE tiene un enfoque más centrado en la legitimidad de input, con reformas institucionales y énfasis en el modelo de gobernanza. Ante la crisis de confianza en la UE propone su democratización, que incluye la necesidad de una «autoridad ejecutiva única creíble» y con plena legitimidad democrática, el sistema Spitzenkandidat y listas transnacionales, más poder para el PE de control e iniciativa, el desarrollo de un Tesoro y presupuesto común e, incluso, la mejora de procedimientos de control democrático de los EE. MM. a través del art. 7 del Tratado (ver Closa, 2018 sobre su aplicación y limitaciones). Es decir, un modelo de gobernanza de Estado Federal. El PE enfatiza el aspecto democrático, de rendición de cuentas y transparencia, poniendo en valor su posición como institución sede de la soberanía popular en la UE. Critican por ello la adopción de «instrumentos intergubernamentales fuera del marco jurídico de la Unión", como el MEDE o el Pacto Presupuestario (Tratado de Estabilidad, Coordinación y Gobernanza en la UEM) y la propensión ya mencionada a crear nuevas instituciones, como ya señaló De Wilde (2019).

Derivado de estos dos enfoques, se deducen las respectivas posiciones sobre la reforma de los Tratados. La Comisión considera que el actual 
entramado institucional es suficiente para llevar a cabo sus propuestas de reforma. Por ejemplo, destacan la posibilidad de utilizar las denominadas pasarelas que contempla el Tratado de Lisboa y que permiten el paso a la mayoría cualificada en la toma de decisiones. Esta posición, como veremos, es compartida expresamente por diversos líderes políticos nacionales. En cambio, el PE presenta la postura contraria y considera que los cambios propuestos requieren de una reforma de los Tratados.

Vemos así el conflicto entre las instituciones europeas, la politización "arriba» a la que se refería Schmidt, y cómo la policrisis europea ha supuesto un cambio en las relaciones de poder y en el modo de concebir las posible reformas políticas e institucionales para abordarlas. De fondo, podemos observar dos modos distintos de encarar la crisis europea: uno se centra en los resultados tangibles para los ciudadanos a través de mejores políticas con un mayor impacto real en la ciudadanía mientras que el otro, en cambio, incide en la necesidad de reformas institucionales para mejorar la legitimidad de origen y hacer más democrático y transparente el propio sistema político. En cierto sentido, ambas instituciones defienden la posición que más se acerca a sus propias atribuciones y legitimidad. Ahora bien, ¿cómo defienden los líderes políticos nacionales?

\section{EL DEBATE DE LOS JEFES DE ESTADO Y DE GOBIERNO}

La tabla 2 presenta de modo esquemático la posición atribuida a cada líder político según el análisis de su intervención, así como la región europea.

De manera general, varios aspectos son reseñables en la comparación entre las intervenciones de los líderes políticos. En primer lugar, todos expresan con claridad qué políticas debería llevar a cabo la UE. Existen diversas posiciones, pero todos le dedican tiempo y argumentos para desgranar sus propuestas. Esto, en cambio, no ocurre con respecto al modelo de gobernanza ni a la posible reforma de los Tratados. Vemos, pues, que el régimen de políticas es central para todos los líderes políticos, anticipándonos una posición más cercana a la legitimidad del ouput y, además, no se han observado posiciones que defiendan una creciente nacionalización de las políticas europeas. En segundo lugar, parecen emerger, con todas las cautelas, ciertas posiciones comunes según la región europea. Por ejemplo, Europa Occidental y del Norte agrupan el grueso de posiciones favorables a la focalización de las políticas europeas, en búsqueda del valor añadido europeo, siguiendo la lógica instrumental señalada por Schimmelfenning y Winzen (2020). Mientras, son los países del Sur de Europa los que defienden un modelo más federal de Unión para, también, ser capaces de gestionar la creciente falta de legitimidad 
Tabla 2. La posición de los lideres politicos

\begin{tabular}{|c|c|c|c|c|}
\hline & $\begin{array}{c}\text { Jefes de Estado y/o } \\
\text { Gobierno }\end{array}$ & $\begin{array}{l}\text { Régimen de } \\
\text { políticas }\end{array}$ & $\begin{array}{l}\text { Modelo de } \\
\text { gobernanza }\end{array}$ & $\begin{array}{l}\text { Reforma } \\
\text { Tratados }\end{array}$ \\
\hline \multirow{9}{*}{$\begin{array}{l}\text { Europa } \\
\text { Occidental } \\
\text { y del Norte }\end{array}$} & Emmanuel Macron (FRA) & + & UF & $S / R$ \\
\hline & Charles Michel (BEL) & + & CG & No \\
\hline & Xavier Bettel (LUX) & + & CG & No \\
\hline & Mark Rutte (PB) & $+/-$ & GD & No \\
\hline & Angela Merkel (ALE) & + & CG & $\mathrm{S} / \mathrm{R}$ \\
\hline & Lars Løkke Rasmussen (DIN) & $+1-$ & CG & $S / R$ \\
\hline & Juha Sipilä (FIN) & $+1-$ & $\mathrm{S} / \mathrm{R}$ & S/R \\
\hline & Stefan Löfven (SUE) & $+/-$ & G & S/R \\
\hline & Leo Varadkar (IRL) & + & UF & $S / R$ \\
\hline \multirow{4}{*}{$\begin{array}{l}\text { Sur de } \\
\text { Europa }\end{array}$} & António Costa (PT) & + & UF/CG & No \\
\hline & Alexis Tsipras (GRE) & + & $\mathrm{EF}$ & $\mathrm{Si}^{*}$ \\
\hline & Pedro Sánchez (ESP) & + & EF & $\mathrm{S} / \mathrm{R}$ \\
\hline & Giuseppe Conte (IT) & + & $\mathrm{EF}^{*}$ & $\mathrm{Si}^{*}$ \\
\hline \multirow{7}{*}{$\begin{array}{l}\text { Europa } \\
\text { Central y } \\
\text { Oriental }\end{array}$} & Andrej Plenković (Croacia) & + & GD & No \\
\hline & Mateusz Morawiecki (POL) & + & GD & $S / R$ \\
\hline & Jüri Ratas (EST) & + & $\mathrm{CG}^{*}$ & No \\
\hline & Klaus Werner Iohannis (RUM) & + & $S / R$ & No \\
\hline & Nicos Anastasiadis (CHIPRE) & + & $\mathrm{S} / \mathrm{R}$ & $\mathrm{S} / \mathrm{R}$ \\
\hline & Peter Pellegrin (ESLOV) & $+/-$ & CG & $\mathrm{S} / \mathrm{R}$ \\
\hline & Krišjānis Kariņš (LET) & $+1-$ & S/R & $S / R$ \\
\hline
\end{tabular}

Régimen de políticas: + (Más comunitarización) y +/- (Focalización).

Modelo de Gobernanza: GD (Gobernanza Dual), CG (Club de Gobernanza), EF (Estado Federal) y UF (Unión Federal).

Reforma de los Tratados, Sí (a favor), No (en contra). En todos los casos, S/R (Sin Referencia).

* Sin referencia explícita pero la posición se desprende de la lógica de su argumento.

Fuente: elaboración propia.

y desafección de los ciudadanos europeos con respecto a la UE y, quizá, también internamente. Por último, en línea con lo propuesto por De Wilde (2019), vemos una posición contraria a la reforma de los Tratados en el grueso 
de los líderes políticos que expresa su posición. Veamos, a continuación, cuáles son los argumentos que se proporcionan en cada tema concreto.

\section{EL RÉGIMEN DE POLÍTICAS}

Todos los líderes políticos presentaron sus propuestas sobre qué políticas debe desarrollar la UE en el futuro. Estas incluyen profundizar la política comercial, aplicar el Acuerdo de París, políticas concretas para desarrollar el pilar social, la creación de empleo, innovación, crecimiento y prosperidad. Existe también un amplio acuerdo sobre la necesidad de finalizar la unión económica y monetaria, la unión bancaria, fortalecer FRONTEX, colaborar en inmigración, desarrollar el mercado digital, la unión energética y la cooperación reforzada permanente en defensa (PESCO).

Esta visión compartida sobre las líneas generales de actuación política se deriva de una concepción similar sobre desafíos y amenazas a los que se enfrenta la UE, como son las migraciones, el cambio climático, la crisis del multilateralismo, la relación con las principales potencias globales (especialmente con China, Rusia y EE. UU.), el terrorismo y las ciberamenazas. A pesar de este acuerdo general, podemos distinguir dos posiciones con respecto al régimen de políticas (ver tabla 2). Por un lado, una mayoría de jefes de Estado y Gobierno abogan por una mayor comunitarización de políticas a nivel europeo, mientras que otros (especialmente los países del Norte de Europa, aunque no solo) prefieren priorizar determinadas políticas. Por tanto, más allá de una posición concreta sobre el desarrollo específico de una política, lo que viene a enfatizar esta distinción es el debate y posiciones diferenciadas sobre en qué debe centrarse las políticas de la UE, teniendo en cuenta los recursos disponibles limitados. Es necesario enfatizar que, al menos entre los líderes intervinientes y en este debate en concreto, no existen posiciones claramente favorables a una nacionalización general de las políticas europeas.

Para ello, y puesto que estos debates coincidieron en el tiempo con la negociación del marco financiero plurianual (MFP, 2021-2027), nada mejor que centrarse en la visión sobre el presupuesto para comprender mejor estas propuestas. Como se pregunta el primer ministro eslovaco Peter Pellegrin, «¿cómo podemos hablar sobre el nuevo MFP sin tener una visión clara de la Unión Europea?». La discusión sobre el presupuesto y el régimen de políticas están intrínsecamente ligados a una determinada visión sobre el futuro de Europa.

El debate sobre el MFP supone dotar de financiación concreta a las políticas. Las distintas posiciones en las diversas regiones y países emergen de manera clara. Como hemos comentado, tenemos países contribuidores y beneficiaros netos del presupuesto, países y regiones que reciben más o menos 
fondos de políticas supranacionales de la UE (cohesión y PAC), y países que debido a sus propios intereses nacionales priorizan determinadas políticas sobre otras. Lo que es poco creíble es defender nuevas y mayores atribuciones europeas manteniendo las políticas tradicionales y sin aumentar el presupuesto. Macron lo expresa claramente al vincular la nueva soberanía europea a un presupuesto financiero que «debe expresar un proyecto político de coherencia, de eficacia y de convergencia». Francia puede aumentar su contribución, pero «es una refundación del presupuesto mismo que es necesario pensar, creando nuevos recursos propios, yo soy favorable, sobre lo digital como sobre ciertos recursos energéticos». En definitiva, el régimen de políticas (y su cobertura presupuestaria) es un modo —el preferente para los líderes políticos— de definir cómo debe ser el futuro de Europa.

Ante esta situación, los países que optan por mantener controlado el presupuesto europeo abogan necesariamente por una priorización de las políticas. Esto va en dos líneas. Por un lado, qué es lo que la UE debe realmente hacer. El primer ministro sueco Löfven es claro pidiendo un presupuesto controlado, no más alto que el actual, y «la priorización más fuerte de la innovación, los nuevos puestos de trabajo y la infraestructura digital, educativa y física necesaria para hacerlos posibles». Unido a esto, el primer ministro de los Países Bajos, Mark Rutte, plantea la necesidad de reducir el presupuesto por la salida del Reino Unido, un contribuyente neto, y critica el excesivo gasto en la PAC y fondos estructurales puesto que si se gastara menos en estas partidas, se dispondrían de mayores recursos para financiar las nuevas prioridades del mañana. En una línea similar se expresa el premier letón Karins, defendiendo mayor inversión en investigación y desarrollo, trabajo, crecimiento, cohesión (aunque de manera inteligente) y critica la PAC. El primer ministro belga Charles Michel enfatiza la necesidad de gastar mejor, con un control del presupuesto comunitario similar al de los nacionales.

En cambio, los países más beneficiados de las políticas tradicionales de la UE son aquellos que defiende la necesidad de un presupuesto más ambicioso. Esto es claro en el Sur de Europa, donde los líderes de España, Italia y Portugal abogan por incrementar el importe total del presupuesto europeo, dotándolo incluso con más recursos propios y, en todo caso, manteniendo un equilibrio entre las políticas tradicionales y las nuevas. Mientras que los países del Norte de Europa defienden su idea del futuro de Europa en una serie de políticas, así también lo justifican los países del Sur. Como indica el propio Pedro Sánchez, «reducir el presupuesto implica aceptar la idea de que Europa se retira, de una Europa en retroceso». Pero también otros beneficiarios netos de estas políticas defienden su importancia. El más claro es el polaco Morawiecki, y vemos aquí también un discurso en clave nacional, al comentar que "los intentos de reducir la política de cohesión son populismo». 
Por tanto, existe un gran potencial de politización con respecto al régimen de políticas de la UE del futuro, especialmente sobre qué se financia y cómo. Algunos países apuestan por una mayor capacidad propia de la UE para dotarse de recursos propios -España, Francia, Polonia, Italia, Grecia, Alemania - frente a aquellos que quieren controlarlo más y, como comenta el primer ministro irlandés Leo Varadkar, tiene una visión fuerte sobre los impuestos nacionales que financian los presupuestos nacionales y que deben ser fijados por los Parlamentos y Gobiernos nacionales. La fractura entre acreedores y deudores, entre el Norte (en un sentido extendido) y el Sur y ciertos países de Europa Central y Oriental, muestran cómo el futuro de Europa tiene matices, aunque existan prioridades políticas compartidas.

De fondo, además, hay una idea importante. Diversos líderes, no por casualidad del Norte de Europa, abogan por una Unión más eficiente, que haga más de lo que promete y que aporte valor añadido a los EE. MM. Vemos aquí un matiz importante con respecto al debate sobre el futuro de Europa. Como en algún escenario propuesto por la Comisión Europea, la UE debería centrarse en aquello que mejor hace y que mayores beneficios aporta a sus EE. MM. y, por lo tanto, la idea de más Europa no es la solución a la policrisis. En palabras de la canciller Angela Merkel, «no todos los problemas en Europa son problemas para Europa».

Podemos observar, finalmente, la lógica del disenso limitante (Hooghe y Marks, 2018) y la importancia de la idea del framing nacional de las debates europeos. Al estilo de la Comisión, el foco principal se basa en qué debe hacer la UE y, en el caso de los líderes nacionales, esto está íntimamente ligado al interés general estatal y la lógica del intergubernamentalismo discursivo (De Wilde 2019). Los impactos diferenciados de las sucesivas crisis europeas no han hecho más que ahondar en ello, fomentando las posibilidades de politización de una manera muy visible, especialmente si nos centramos en qué políticas potenciar, cómo se financian y su impacto sobre el alcance de la UE. Unido a esto, adquiere especial relevancia no solo el régimen de políticas, sino también el modelo de gobernanza, como veremos en el siguiente apartado.

\section{EL MODELO DE GOBERNANZA O CÓMO ORGANIZAR LA UE DEL FUTURO}

De la discusión anterior se desprenden algunos aspectos que nos muestran que, a pesar del foco de muchos actores e instituciones se centra en las políticas, los arreglos institucionales también son importantes.

Fabbrini (2019) señaló dos principios básicos sobre el modelo de gobernanza donde la UE puede verse como una organización regional o, en cambio, como una federación en construcción. Como hemos indicado, entra en juego 
la idea de diferenciación en el propio proceso de integración europea, con una UE de varias velocidades, a la carta, con diversos países que eligen el nivel de integración que más les interesa o, en cambio, la idea de una mayor uniformidad donde todos los EE. MM. estén, si cumplen los requisitos necesarios, en todas las políticas europeas.

Este modo de conceptualizar el debate sobre el futuro de Europa está ejemplificado por la posición del PE, derivada de sus dos resoluciones al respecto. Sin negar la importancia de qué debe hacer la UE, es igualmente importante establecer cuáles son los arreglos institucionales, quién decide, quién es responsable y cómo se toman las decisiones, así como las consideraciones relativas a la calidad democrática, la rendición de cuentas y la transparencia de la propia Unión. Asimismo, el modo de enfrentar las policrisis y el creciente distanciamiento ciudadano con respecto a la UE no se debe en exclusiva a la percepción de los malos resultados en determinadas políticas y la capacidad de reacción de la UE en diversos ámbitos, sino también está ligado a los problemas de diseño institucional, de complejización en la toma de decisiones y de la percepción sobre la falta de legitimidad de algunas de las principales instituciones europeas. Frente a la legitimidad del output también es importante la legitimidad del input.

Esto, incluso, ha dado pie a nuevas teorías sobre el euroescepticismo. Como indica De Vries (2018) en su teoría de la referencia (benchmark theory), la posición de los ciudadanos con respecto al proceso de integración se establece comparando la evaluación de las políticas y el propio régimen nacional y europeo. Esta teoría pone de manifiesto cómo los ciudadanos de aquellos países que mejor evalúan su propio sistema pueden más críticos ser con respecto a la propia UE, aunque su evaluación de las políticas europeas y el sistema europeo sea positiva. Por el contrario, aquellos ciudadanos que evalúan peor las políticas de su propio país con respecto a la UE están más abiertos a compartir una mayor soberanía con un sistema que valoran mejor que el propio.

La codificación de las posiciones de los líderes, con todas las cautelas y limitaciones anteriormente señalados, parece ratificar esta idea. Los representantes de los países del Sur de Europa, y quizá el propio presidente Macron y el irlandés Varadkar, son aquellos que defienden, algunos de modo explícito, la idea de una UE federal. Es claro en la intervención de Pedro Sánchez, que indica la necesidad de apostar por la soberanía europea y cómo «proteger Europa es avanzar - como decía - en la Europa social, en la Europa de la defensa y la unión económica y monetaria. Una Europa, en definitiva, federal, que se relegitima si conquista el favor de su ciudadanía [...]». Esta idea de la soberanía europea es central también en la posición del presidente Macron. La idea de democracia y sus valores es central en Europa y, por ello, ante las 
amenazas a la misma, "podemos y debemos construir una nueva soberanía europea con la que aportemos una respuesta clara y firme a nuestros ciudadanos que podemos protegerles y proporcionar una respuesta a los desórdenes del mundo». Esta soberanía europea necesita el desarrollo de este demos europeo, como indica el italiano Giuseppe Conte, y supone la profundización de la unificación política, con instituciones europeas más fuertes y democráticas, en palabras de Tsipras. Esto implica un Parlamento más fuerte, una rendición de cuentas más claras y, en definitiva, la construcción de una federación. Ahora bien, quizá podamos observar una diferencia entre las propuestas federales. Mientras los tres grandes países del Sur, que han sufrido especialmente la crisis económica y de la inmigración, parecen proponer un desarrollo institucional que lleve a un Estado federal, en Macron se atisba una idea de unión federal, donde frente a los grandes cambios, retos y transformaciones mundiales, "tenemos necesidad de una soberanía más fuerte que la nuestra, complementaria y no de substitución [de la nacional]». En esta línea se expresa también Leo Varadkar, que defiende la democratización en la elección de los principales cargos de la UE, el sistema de spitzenkandidat y las listas transnacionales. Su propuesta cabría incluirla dentro de la lógica de unión federal, puesto que como él mismo señala muchos estados de EE. UU. y provincias de Canadá tienen más autonomía y variación entre ellos que los propios EE. MM. de la UE. En esta lógica defiende también la idea de diferenciación, de la que Irlanda se beneficia con su política de impuestos corporativos, puesto que no todo debe estar armonizado y estandarizado.

Frente a esta modelo de gobernanza, podemos distinguir también propuestas que conciben la UE como una organización regional. El grueso de los representantes de los países noroccidentales y de Europa Central y Oriental van en esta línea, aunque con distintas posiciones. Parece existir una relación entre qué debería hacer la UE, especialmente si esta debe focalizarse en determinadas políticas que aporten valor ańadido a los EE. MM., y las preferencias sobre el modelo de gobernanza. Un claro ejemplo es la posición del neerlandés Mark Rutte, que propone unos Estados soberanos trabajando juntos para la prosperidad, seguridad y estabilidad. No está a favor de la diferenciación ni de la Europa a la carta. El debate se aleja de la dicotomía "más o menos Europa», para centrarse en dónde aporta valor y el necesario equilibrio entre los Parlamentos nacionales y el Parlamento europeo. Parece establecer una idea de gobernanza dual, puesto que como veremos en el siguiente apartado, defiende la idea de que la UE ya dispone de las herramientas suficientes para su funcionamiento y adaptación, aunque no se están utilizando adecuadamente.

Esta lógica de organización regional y gobernanza va, por tanto, íntimamente ligada al papel de las instituciones nacionales. Así lo defienden los representantes polaco y croata, por ejemplo. Como indica Mateusz 
Moraviecki, son los Parlamentos nacionales los que representan al soberano y son la base de la democracia. La crisis de la UE, también, se debe a la percepción ciudadana de pérdida de control con respecto a las crecientes competencias de determinadas instituciones europeas y, por tanto, es necesario redefinir el equilibrio entre Estados nacionales y la cooperación a nivel europeo. En sus propias palabras, «la nación de naciones 2.0», al estilo de De Gaulle. Frente a la lógica federal, los problemas de legitimidad democrática de la Unión se solucionan a nivel nacional, no creando un superestado europeo.

Otros líderes políticos, dentro de la lógica de la gobernanza, defienden ideas más cercanas al club de gobernanza. En este sentido, es clave la idea de diferenciación y la Europa de varias velocidades. Como hemos visto, se justifica teniendo en cuenta la creciente diversidad entre los EE. MM., que hace difícil, para algunos desaconsejable, que todos deban, puedan e incluso quieran, participar en todas las políticas al mismo tiempo. El primer ministro belga, Charles Michel, indica cómo el nivel nacional y europeo deben coordinarse, complementarse y reforzarse. Los EE. MM. deben contar con solidaridad compartida, pero también con su propia responsabilidad individual, y defiende la idea de una Europa de varias velocidades, justificada en la lógica de que todos los avances europeos siempre han provenido de una vanguardia. Aunque, matiza, está en contra de una Europa a la carta. Esta visión está en línea con uno de los escenarios del Libro blanco de la Comisión, que establecía que "los que desean hacer más, hacen más», la lógica instrumental y la integración diferenciada. Por tanto, ciertos EE. MM. pueden avanzar en aquellas políticas que consideren necesarias y más valor les aporte. Este enfoque es similar en otros líderes políticos como Xavier Bettel o Juha Sipilä. Esta diversidad que permite la diferenciación también es aplicable dentro de otra lógica. Como indica el primer ministro António Costa, su posición es contraria a la uniformidad, abogando por la libertad de los EE.MM para llevar a cabo sus propias políticas para cumplir los objetivos y acuerdos europeos. Esto adquiere relevancia teniendo en cuenta que su Gobierno implementó unas políticas contrarias a ciertas propuestas relacionadas con la austeridad, defendidas por las instituciones europeas e internacionales pero logrando, aun así, los objetivos macroeconómicos establecidos. En este caso, la diferenciación permite un mayor margen de maniobra político a los Gobiernos en el desarrollo de sus propios programas y prioridades nacionales.

Esta idea de la diferenciación no es compartida por todos. De hecho, los países de Europa Central y Oriental son aquellos que más contrarios se muestran, al temer ser excluidos de los avances. En esta línea se expresan los líderes de Rumanía y Estonia. Como indica Klaus Werner Iohannis, se necesita una Unión que no deje atrás a ningún Estado, junto con la necesidad de mantener la unidad e indivisibilidad de la UE, incompatible con las varias velocidades o 
los círculos concéntricos. El estonio Júri Ratas apunta a otro aspecto, tratado también en el sexto escenario presentado por Juncker, sobre la necesidad de que aquellos países que cumplan con los criterios fijados pasen a formar parte de los distintos acuerdos europeos. Al igual que el presidente de la Comisión, que señalaba cómo el euro es la moneda de la Unión y todos deberían estar en ella en cuanto cumplieran los requisitos, así lo indica el primer ministro estonio al lamentar que existan miembros que no formen parte del acuerdo de Schengen aunque cumplan todo los criterios.

Existe un aspecto final interesante, pero que complica la determinación de las posiciones y nos indica la diversidad de ideas sobre qué debe ser la UE, las diferencias de fondo de cada modelo de gobernanza y la posibilidad de que este aspecto fomenta la politización del debate. Aunque casi todos los líderes políticos destacan la importancia de la unidad europea, esta adquiere distintos significados y matices. Para algunos, la unidad se refuerza con la construcción de la unión política, mientras que para otros esta unidad no significa uniformidad y es necesario conocer y respetar la diversidad de cada país y sus circunstancias. Una serie de factores nacionales juegan un papel central en esta posición, más aún cuando se trata de la posición de sus principales líderes políticos (De Wilde, 2019).

\section{3. ¿̇ES NECESARIA UNA REFORMA DE LOS TRATADOS?}

Un último aspecto merece ser analizado. Tanto el régimen de políticas como el modelo de gobernanza deben tener encaje en los Tratados que regulan el funcionamiento de la propia UE. Teniendo en cuenta las policrisis que ha experimentado la Unión, junto con la creciente crítica ciudadana sobre su funcionamiento, ciertas propuestas de cambio o evolución profunda requerirían, cuanto menos, de una adaptación de los Tratados. Se puede plantear la relación entre las propuestas sobre el modelo de gobernanza y la necesidad o deseabilidad de la reforma de los Tratados. Según esto, aquellos líderes que defienden un modelo de gobernanza de tipo federal, que requiere aumentar la soberanía compartida y fortalecer políticamente las instituciones comunes, deberían optar por una reforma de los Tratados, puesto que estas propuestas difícilmente tienen encaje en la configuración actual. Y viceversa. De hecho, estas dos posiciones quedan reflejadas en la posición del PE y la Comisión, respectivamente.

Con respecto al debate de los jefes de Estado y/o Gobierno es necesario incluir otro aspecto fundamental: el proceso de cambio y aprobación de los Tratados es largo y complejo. Además, puede tener un coste político enorme en el ámbito nacional, puesto que en muchos casos requiere de referéndums nacionales para su aprobación — con resultados, desde el año 2000, no 
favorables a las posiciones prointegración europea- ${ }^{8}$. En esta lógica, De Wilde (2019: 1204) señala que este proceso, que necesariamente adquiere relevancia mediática al ser una referéndum de ámbito estatal y visibilidad europea, puede afectar a los compromisos a los que llegan los líderes y, por tanto, tenderán a evitar las reformas de los Tratados, donde prima además una lógica eminentemente nacional.

Con todas las cautelas expresadas anteriormente, y más aun teniendo en cuenta que hasta diez líderes no se posicionan claramente sobre este tema, esta hipótesis parece sustentarse en nuestro análisis. Todos los que se oponen de manera expresa a la reforma de los Tratados defienden la idea de gobernanza europea. Esto es evidente en sus argumentos. En la lógica de mantener o ahondar en la actual arquitectura institucional, se defiende que los Tratados proporcionan las herramientas necesarias para su actuación, pero el problema es que no se cumplen sus disposiciones. En esta línea, como vimos, la Comisión proponía utilizar las pasarelas disponibles en el Tratado de Lisboa para pasar de la unanimidad a mayoría cualificada en la toma de decisiones. Mark Rutte seńala la querencia de la UE a crear nuevas normas y acuerdos cuando ni siquiera se cumplen los pactos actuales (De Wilde, 2019: 1204) señala esta predisposición a crear instituciones de novo. Por ejemplo, Rutte se opone a las transferencias directas en la eurozona porque con el Pacto para la Estabilidad y el Crecimiento ya es suficiente, siempre que se cumpla. Adicionalmente, para algunos líderes la reforma de los Tratados siempre ha supuesto más Europa en detrimento de los Estados, como demuestra la historia de la integración europea. Un tercer argumento señala la relación entre régimen de políticas y la no reforma: este proceso restaría energía para centrase en lo relevante, el régimen de políticas, puesto que, en palabras de António Costa, "la arquitectura institucional es solo un instrumento que nos permitirá su concreción. Invertir el orden es contraproducente y nos divide en el accesorio antes de unirse sobre lo esencial». Por último, y no menos importante, algunos señalan cómo los ciudadanos sienten estos debates institucionales como lejanos y carentes de interés, restando energías para los temas realmente importantes. De nuevo, se desprende una idea de legitimidad de ouput.

Entonces, aquellos líderes que más han enfatizado que el problema de legitimidad de la UE se debe no solo a sus políticas, sino al modo en que estas se adoptan y la falta de legitimidad y democracia a nivel europeo, son los que promulgan la necesidad de la reforma de los Tratados, unida a la idea de un

8 Hix y Holand (2012) señalan que, desde el año 2000, de los dieciséis referéndums llevados a cabo en la UE, solo en cinco ganaron las posiciones prointegración. 
modelo de gobernanza de estilo federal. Esto es cierto, de manera más o menos explícita, en las intervenciones de los líderes italiano y griego, dos de los países que más han sufrido las consecuencias nacionales de la toma de decisiones europeas, tanto en la crisis económica como en la migratoria y de refugiados. En esta línea, Giuseppe Conte señala las críticas a las limitaciones de la arquitectura institucional europea y la necesidad de que «con coraje y con rigor [debemos] empeñarnos para que se reconsidere aquello que no ha funcionado, también en la implementación de los tratados [...]", para así poder encarar las tendencias hacia la desintegración y desilusión europea, como ocurre en Italia. La lógica federal de Tsipras le lleva a defender reformas audaces en democracia, transparencia y justicia. Vemos así, también, una estrategia de protección de los intereses nacionales desde la óptica de la defensa de una serie de principios democráticos, en línea con la hipótesis de desbordamiento democrático de De Wilde (2019: 1198-1199).

En conclusión, la discusión sobre la idoneidad de reformar los Tratados manifiesta cómo existen, de fondo, diversas posiciones sobre cómo debe ser el futuro de Europa, sus necesidades y prioridades y el modo de llevarlo a cabo.

\section{CONCLUSIÓN}

El análisis comparado de las propuestas de las instituciones europeas y de los principales líderes políticos nacionales nos muestra el potencial para la creciente politización del tema europeo en diversos niveles y señala la importancia de centrarnos en el régimen de políticas y en el modelo de gobernanza. Ambos están interrelacionados y definen, también, la posición sobre la posible reforma de los Tratados. El estudio de las diferentes propuestas proporciona argumentos sobre algunas de las hipótesis planteadas por diversos autores sobre el impacto diferencial de la policrisis según la región europea, el papel del disenso limitante y la lógica nacional, el peso del intergubernamentalismo discursivo y las lógicas de integración diferenciada y sus consecuencias. Varios aspectos adicionales merecen ser destacados.

En primer lugar, la escasa interacción entre las propuestas de las instituciones europeas y los líderes políticos nacionales. Muy pocos entre ellos hicieron referencia a las propuestas concretas elaboradas por la Comisión y el Parlamento (Drachenberg y Kotanidis, 2019). Vemos, de nuevo, la idea del disenso limitante (Hooghe y Marks, 2009), la lógica de politización de «abajo a arriba» que señaló Schmidt (2019a) y la preeminencia del intergubernamentalismo discursivo (De Wilde, 2019). La politización entre diversos niveles no viaja bien, pudiendo afectar al tipo de debate - de preeminencia nacional e intereses particulares- sobre el futuro de la integración. 
En segundo lugar, a pesar de llamamientos genéricos a la unidad y la importancia de mejorar la UE para responder a sus desafíos, se observan dos posiciones de fondo contrapuestas, que tienen que ver con el modo de adaptarse a las causas de la crisis europea. Para unos, como la Comisión y muchos líderes políticos, esta se debe a un problema de legitimidad de output y, por tanto, se centran en el régimen de políticas. Para otros, con el PE y los líderes del Sur de Europa como máximos exponentes, se trata también de un problema institucional y de legitimidad de input. Esto va unido al impacto diferencial de las sucesivas crisis europeas según el país y región, y su consecuente influencia a la hora de proponer y reflexionar sobre la Europa del futuro. El impacto diferencial hace que las prioridades y propuestas políticas sean distintas y puede tener un efecto profundo en las posiciones y negociación sobre el futuro de Europa.

En tercer lugar, aunque se pueden establecer preferencias comunes entre determinados países y regiones, estos grupos no son monolíticos y difieren en ciertas prioridades y propuestas, facilitando también la negociación y el debate. Esto puede ayudar a que la politización sirva para llegar a acuerdos más que a complicarlos. Existe margen de negociación y posiciones matizadas.

En cuarto lugar, y es interesante en el momento actual, vemos que no existen propuestas claras de salida de la UE por parte de los líderes más críticos, aunque es cierto que alguno de ellos no participó en el debate. Ciertos partidos en determinados países puedan hacer propuestas a este respecto, pero en los debates analizados se observan visiones distintas, diversas preferencias sobre el equilibrio entre niveles o las competencias que debe tener la UE, incluido un mayor peso estatal. Pero, en ningún caso, el fin explícito del proceso de integración. A pesar de las policrisis, la creciente desafección y el auge de discursos euroescépticos, la UE muestra resiliencia. Habrá que ver cómo evolucionan o se clarifican estas posiciones si la politización «abajo», con actores nacionales y opiniones públicas más críticas, comienza a activar el tema según el proceso vaya ganando relevancia.

Finalmente, vemos como la politización multinivel es cada vez más relevante, pero falta, y es un aspecto crucial, incluir a los ciudadanos en la discusión. En este sentido, es interesante destacar la reflexión del primer ministro danés Rasmussen, que comentaba que en su país tienen «una larga tradición de discutir sobre la Unión Europea. [...] Esto nos ha dado a todos, incluido a mí mismo, una visión más pragmática de nuestra Unión». Con esta politización y el conocimiento que de ella se deriva, su posición es mucho más funcional y menos idealista. Esta fractura entre proyectos ideales y su visión práctica es, quizá, el reto que tiene por delante la cristalización de los debates sobre el futuro de Europa. 
Esta posible politización y el papel de los ciudadanos son dos aspectos cruciales en los siguientes pasos en el debate sobre el futuro de Europa. La Comisión von der Leyen presentó el 22 de enero de 2020 su propuesta para organizar los trabajos de esta conferencia y las críticas no han tardado en llegar, por su modelo jerárquico y el escaso papel ciudadano. Controlando el tipo de debates y reduciendo los espacios deliberativos, las instituciones de la UE, de nuevo, "corren el riesgo de aumentar las expectativas que luego no pueden fácilmente cumplir» (Alemanno, 2020), produciendo una mayor erosión de la confianza ciudadana. Esta idea de cómo la UE promete mucho, pero no consigue ofrecer resultados consistentes, es un argumento frecuente en los líderes políticos. La capacidad de establecer un proceso creíble, de tener una discusión clara y mecanismos para integrar a los ciudadanos en la misma, será crucial para el resultado del debate y mejorar la legitimidad de la propia UE. De esto, en el fondo, trata el debate sobre el futuro de Europa, con posibles y profundas consecuencias.

\section{Bibliografía}

Alemanno, A. (2020). The conference on the Future of Europe: An open letter. Verfassungsblog [blog], 01-02-2020. Disponible en: https://bit.ly/32Mskxq.

Börzel, T. y Risse, T. (2009). Revisiting the nature of the Beast. Politicization, European integration and postfuncionalism: A comment on Hooghe and Marks. British Journal of Political Science, 39 (1), 217-220. Disponible en: https://doi.org/10.1017/ S000712340800046X.

Closa, C. (2018). La protección de los valores fundamentales de la UE frente a la violación por parte de sus estados miembros. En C. Closa e I. Molina (coords.). El Futuro de la Unión Europea (pp. 93-104). Madrid: Real Instituto Elcano.

Comisión Europea (2017). Libro blanco sobre el futuro de Europa. Reflexiones y escenarios para la Europa de los veintisiete en 2025.

Consejo Europeo (2019). Declaración de Sibiu. Comunicado de Prensa 335/19. Disponible en: https://bit.ly/3iU9iLe.

De Vries, C. (2018). Euroscepticism and the future of European Integration. Oxford: Oxford University Press. Disponible en: https://doi.org/10.1093/oso/9780198793380.001.0001.

— y Edwards, E. E. (2009). Taking Europe To its extremes extremist parties and public euroscepticism. Party Politics, 15 (1), 5-28. Disponiblen en: https://doi. org/10.1177/1354068808097889.

De Wilde, P. (2019). Media logic and grand theories of European integration. Journal of Euorpean Public Policy, 26 (8), 1193-1212. Disponible en: https://doi.org/10.1080/135017 63.2019.1622590.

— , Leupold, A. y Schmidtke, H. (2016). Introduction: the differentiated politicisation of European governance. West European Politics. 39 (1), 3-22. Disponible en: https://doi. org/10.1080/01402382.2015.1081505. 
Drachenberg, R. y Kotanidis, S. (2019). Los debates sobre el futuro de Europa en el Parlamento Europeo (2018-2019). European Parliament Research Service. Disponible en: https:// bit.ly/3iQvaqK.

Fabbrini, S. (2019). Alternative governance models: «Hard core» in a differentiated Europe. Comparative European Politics. 17 (2), 278-293. Disponible en: https://doi.org/10.1057/ s41295-019-00158-5.

- y Schmidt, V. (2019). Introduction: differentiation and beyond. Comparative European Politics. 17 (2): 173-175. Disponible en: https://doi.org/10.1057/s41295-019-00163-8. Hix, S. y Hoyland, B. (2012). El sistema politico de la Unión Europea. Madrid: McGraw Hill. Hobolt, S. (2014). A vote for the President? The role of Spitzenkandidaten in the 2014 European Parliament elections. Journal of European Public Policy. 24 (10), 1528-1540. Disponible en: https://doi.org/10.1080/13501763.2014.941148.

Hooghe, L. y Marks, G. (2009). A postfunctionalist theory of European integration: From permissive consensus to constraining dissensus. British Journal of Political Science, 39 (1), 1-23. Disponible en: https://doi.org/10.1017/S0007123408000409.

- (2018). Cleavage theory meets Europe's crises: Lipset, Rokkan, and the transnational cleavage. Journal of European Public Policy, 25 (1), 109-135. Disponible en: https://doi. org/10.1080/13501763.2017.1310279.

— y Wilson, C. J. (2002). Does left/right structure party positions on European integration? Comparative Political Studies 35 (8): 965-989. Disponible en: https://doi. org/10.1177/001041402236310.

Hutter, S. y Kriesi, H. (2019). Politicizing Europe in times of crisis. Journal of European Public Policy, 26 (7), 966-1017. Disponible en: https://doi.org/10.1080/13501763.2019.161 9801.

Jabko, N. y Luhman, M. (2019). Reconfiguring sovereignity: crisis, politicization, and European integration. Journal of Europea Public Policy, 26 (7),1037-1055. Disponible en: https://doi.org/10.1080/13501763.2019.1619190.

Juncker, J. C. (2015). Realizar la unión económica y monetaria. Comisión Europea. Disponible en: https://bit.ly/3iO2gaM.

(2017). Discurso sobre el Estado de la Unión. 13 septiembre de 2017. Disponible en: https://bit.ly/3mOU90e.

Leyen, Ursula von der (2019). Opening statement in the European Parliament plenary session by Ursula von der Leyen, candidate for President of the European Commission. 15 de julio de 2019. Disponible en: https://bit.ly/35ViaMP.

Marks, G., Hooghe, L., Steenbergen, M. R. y Bakker, R. (2007). Crossvalidating data on party positioning on European integration. Electoral Studies 26 (1): 23-28. Disponible en: https://doi.org/10.1016/j.electstud.2006.03.007.

Parlamento Europeo (2017). Resolución del Parlamento Europeo, de 16 de febrero de 2017, sobre posibles modificaciones y ajustes de la actual configuración institucional de la Unión Europea, 2014/2248 (INI).

(2019). Resolución del Parlamento Europeo, de 13 de febrero de 2019, sobre la situación del debate sobre el futuro de Europa, 2018/2094 (INI).

Risse-Kappen, T. (1996). Exploring the nature of the beast: International relations theory and comparative policy analysis meet the European Union. Journal of Common Market 
Studies. 34 (1), 53-80. Disponible en: https://doi.org/10.1111/j.1468-5965.1996. tb00560.x.

Scharpf, F. (2003). Problem-solving effectiveness and democratic accountability in the EU. MPIFG Working Paper, 03/01.

Schimmelfenning, F. y Winzen, T. (2020). Introduction. En F. Schimmelfenning y T. Winzen (eds.). Ever Looser Union? (pp. 1-10). Oxford: Oxford University Press. Disponible en: https://doi.org/10.1093/oso/9780198854333.003.0001.

Schmidt, V. (2019a). Politicization in the EU: between national politics and EU political dynamic. Journal of European Public Policies, 26 (7), 1018-1036. Disponible en: https:// doi.org/10.1080/13501763.2019.1619189.

(2019b). The future of differentiated integration: a «soft-core», multi-clustered Europe of overlapping policy communities. Comparative European Politics, 17 (2), 294-315. Disponible en: https://doi.org/10.1057/s41295-019-00164-7.

Sitter, N. (2002). Opposing Europe: Euro-Scepticism, opposition and party competition. Sussex Euroepan Institute Working Papers, 56.

Taggart, P. (1998). A touchstone of dissent: Euroscepticism in contemporary Western European party systems. European Journal of Political Research, 33 (3), 363-388. Disponible en: https://doi.org/10.1111/1475-6765.00387.

Tilindyte, L. (2019). Election of the President of the European Commission. Understanding the Spitzenkandidaten process. European Parliamentary Research Service. Disponible en: https://bit.ly/32OkaEL.

Van Rompuy, H. (2012). Towards a genuine economic and monetary union. Brussels: European Council.

Zeitlin, J., Nicoli, F. y Laffan, B. (2019). Introduction: the European Union beyond the polycrisis? Integration and politicization in the age of shifting cleavages. Journal of European Public Policy, 26 (7), 963-976. Disponible en: https://doi.org/10.1080/13501763.201 9.1619803.

Zulianello, M. (2019). Varieties of populist parties and party systems in Europe: From stateof-the-art to the application of a novel classification scheme to 66 parties in 33 countries. Government and Opposition, 55 (2), 327-347. Disponible en: https://doi. org/10.1017/gov.2019.21. 